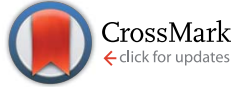

Cite this: RSC Adv., 2017, 7, 13032

Received 12th December 2016 Accepted 11th February 2017

DOI: 10.1039/c6ra28071d

rsc.li/rsc-advances

\section{The rheological characteristics for the mixtures of cationic surfactant and anionic-nonionic surfactants: the role of ethylene oxide moieties $\uparrow$}

\author{
Liming Zhang, ${ }^{a}$ Wanli Kang, ${ }^{\text {*ab }}$ Derong $\mathrm{Xu},{ }^{\mathrm{a}}$ Haishun Feng, ${ }^{\mathrm{a}}$ Pengyi Zhang, ${ }^{\mathrm{a}}$ Zhe $\mathrm{Li}{ }^{\mathrm{a}}$ \\ Yao $\mathrm{Lu}^{\mathrm{a}}$ and Hairong $\mathrm{Wu}^{\star \mathrm{a}}$
}

This study systematically reports the rheological behaviour and mechanism for mixtures of cationic surfactant cetyltrimethyl ammonium bromide (CTAB) and anionic-nonionic carboxylate surfactants (NPEC- $n$ ). The effects of molar ratio, total concentration, salinity, shearing time, temperature, and ethylene oxide (EO) moieties on the microstructures of the mixtures were investigated in detail using rheometry, freeze-fracture transmission electron microscopy (FF-TEM), cryo-transmission electron microscopy (Cryo-TEM), etc. The results indicate that the conformations of the EO moieties concern the head-group areas and steric hindrance, which affect the arrangement of the surfactant molecules. The aggregates with diverse morphologies endow the solutions with different rheological behaviours. Except for the CTAB/NPEC-10 system, the CTAB/NPEC-5 system and CTAB/NPEC-7 system show viscoelastic behaviour under some conditions and their highest viscosities appear at the molar ratio of $76: 24$ and $40: 60$, respectively. The transition temperature of the mixture appears at $35{ }^{\circ} \mathrm{C}$, accompanied with a sharp decrease in the viscosity. The salt thickening and shear-resistant properties of the mixtures have also been discussed, indicating good salt-resistance and shear-resistance of the mixtures.

\section{Introduction}

Mixed surfactant systems have been used in a wide range of applications due to their high surface activity, low critical micelle concentration (CMC), and low interfacial tension as compared to those of single surfactant systems. ${ }^{\mathbf{1 - 1 1}}$ Among these systems, research on cationic/anionic surfactants mixtures is ongoing for a long period of time and has yielded great achievements; the discovery and application of worm-like micelles (WLMs) is an example. ${ }^{12-16}$ WLMs make the mixed solutions present unique properties $;^{17}$ therefore, they have been widely employed in many industries. ${ }^{18-24}$ However, the interactions between the surfactants (e.g. electrostatic repulsion, van der Waals attraction, and hydrogen bonding) function during the process of mixing. ${ }^{25-28}$ It has been reported that the strong interaction between cationic/anionic surfactant molecules makes the mixed solution unstable, such that the precipitation or phase separation occurs when the concentration exceeds the CMC. ${ }^{29}$ For some systems, phase separation even appears at concentrations lower than the CMC, especially in equimolar

${ }^{a}$ Institute of Enhanced Oil Recovery, China University of Petroleum (Beijing), Beijing, 102249, P.R. China.E-mail: kangwanli@cup.edu.cn; hrwu@cup.edu.cn

${ }^{b}$ School of Petroleum Engineering, China University of Petroleum (East China), Qingdao 266580, P.R. China

$\dagger$ Electronic supplementary information (ESI) available. See DOI: $10.1039 / \mathrm{c} 6 \mathrm{ra} 28071 \mathrm{~d}$ solutions. $^{30,31}$ This phase behaviour impedes the practical applications of cationic/anionic systems. It was found that precipitation can be overcome by introducing ethylene oxide (EO) moieties into the hydrophobic chain of some anionic or cationic surfactant molecules. ${ }^{32}$ On the one hand, EO moieties affect the area of the head-group and packing parameter $P ;^{33}$ on the other hand, EO moieties lead to lower charge intensity. Both aspects can effectively reduce the electrostatic interactions between the surfactant molecules. ${ }^{34}$ Furthermore, it is convenient to introduce EO moieties into the molecules of the anionic surfactant; thus, an anionic-nonionic surfactant molecule that displays both the high interfacial activity of anionic surfactant and good salt-resistance of the non-ionic surfactant can be obtained..$^{35}$

The types of anionic hydrophilic head-group and alkoxy groups as well as EO number determine the performance of anionic-nonionic surfactants which include alkoxy sulfates, sulfonates and carboxylates surfactants. Anionic-nonionic surfactants composed of alkoxy sulfates have attracted significant attention. For instance, Hato et $a .^{36-39}$ have studied the effect of EO number on the Kraft point for dodecyl polyoxyethylene ether sulfate, and they found that upon increasing the number of EO moieties, the surface activity is enhanced and the Kraft point is diminished. Minero et al. ${ }^{\mathbf{4 0}}$ reported that the EO moieties play a role in dispersing the charges in the head-group, thus weakening the electrostatic repulsion and promoting the formation of aggregates. By studying the molecular elasticity, 
Masuyama et $a .^{41}$ found that the introduction of EO moieties made the hydrophobic chain of the surfactant more flexible, resulting in an improved surface activity. Chen et al. ${ }^{42}$ explored the micelle formation and synergistic interactions of binary surfactant combinations, which were composed of the synthesized sodium nonylphenol polyoxyethylene ether sulfate (NPES) and various traditional surfactants, indicating that the interaction parameter varied with the composition and the synergistic interaction concurrently existed in the NPES. In contrast, research on carboxylate is relatively rare; Liu et $a l .{ }^{43}$ has reported the effect of electrolytes on the interfacial tension and found that $\mathrm{Na}^{+}$plays a smaller role in reducing the oil/water interface of polyoxyethylene ether carboxylate than that of $\mathrm{Ca}^{2+}$ and $\mathrm{Mg}^{2+}$. To date, research on anionic-nonionic surfactants is mainly focused on the surface activity and micelle formation ability of alkoxy sulfonate surfactants. However, the rheological behaviour and mechanism of mixed anionic-nonionic carboxylate/ cationic surfactant have not been reported to date.

In this study, the cationic surfactant CTAB and three types of carboxylate surfactant (NPEC- $n, n=5,7$, and 10) containing different numbers of EO moieties were mixed together. The mixtures displayed various rheological behaviours on adjusting the molar ratio, total concentration, and EO number. Then, the rheological characteristics, together with the microscopic structures of the aggregates, were combined to analyse the role that EO played in the rheological properties and mechanism. The obtained results contribute to extend the application of carboxylate surfactants as well as provide theoretical guidance for this type of surfactant.

\section{Materials and methods}

\section{Materials}

The quaternary ammonium cationic surfactant cetyltrimethyl ammonium bromide (CTAB, purity > 99\%, A.R. grade) was purchased from Tianjin Fuchen Chemical Reagent Company (Tianjin, China). The anionic-nonionic surfactants NPEC-5, NPEC7, and NPEC-10 with various numbers of EO in the hydrophobic chain were provided by Qingdao Changxing Chemical Co., Ltd. (Shandong, China), and their chemical structures are shown in Scheme 1. $\mathrm{NaCl}$ (>99\%, A.R. grade) was provided by Sinopharm Chemical Reagent Co., Ltd. (Shanghai, China). Water used in solution preparation was pure water without distillation.

\section{Sample preparation}

Samples were prepared by directly mixing CTAB and the three types of carboxylate surfactant solutions in a bottle at various

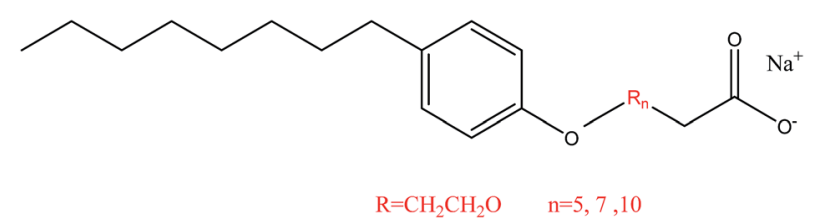

Scheme 1 The chemical structures of NPEC-5, NPEC-7, and NPEC10. molar ratios and molar concentrations. Then, a desired amount of $\mathrm{NaCl}$ was added to the bottle. After sealing, these samples were vortex-mixed and equilibrated at room temperature for $1 \mathrm{~h}$ to ensure complete dissolution and uniformity. The resulting mixtures were stored at $25{ }^{\circ} \mathrm{C}$ in a thermostatic bath for at least $24 \mathrm{~h}$ prior to measurement. All the measurements were performed at $25{ }^{\circ} \mathrm{C}$ unless otherwise specified.

\section{Rheological measurements}

The rheological properties of the samples were measured using a rheometer (HAAKE RS600, Germany). A cone-plate sensor with a plate diameter of $50.00 \mathrm{~mm}$, a cone angle of $1^{\circ}$, and a default gap of $0.052 \mathrm{~mm}$ was used. A chamber for covering the sample was used to avoid evaporation when necessary. The steady state measurement was performed at the shear rate sweep of 0.01$1000 \mathrm{~s}^{-1}$. Dynamic state rheological measurements were carried out at the constant shear stress of $0.1 \mathrm{~Pa}$, which was obtained from the linear viscoelastic region of the frequency sweep measurement.

\section{Transmittance measurements}

The transmittance of the bicomponent surfactant solution was obtained from the scanning curves obtained using a stability analyser (TURBISCAN Lab, France), which was equipped with a pulsed near-infrared light source $(\lambda=880 \mathrm{~nm})$. The samples were placed in a test tube and were scanned from bottom to top at different temperatures. The microscopic properties of the samples were characterized by the scanning curves at different temperatures indirectly.

\section{Freeze-fracture transmission electron microscopy (FF-TEM)}

The morphology of the bicomponent surfactant was observed using a JEOL-100 CX II TEM. First, a small amount of sample was placed on a $0.1 \mathrm{~mm}$ thick copper disk and then covered with a second copper disk. Then, the sample was frozen by plunging into liquid propane, which was cooled using liquid nitrogen. Fracturing and replication were performed using a freezefracture apparatus (BalzersBAF400, Germany) at $-140{ }^{\circ} \mathrm{C}$. Pt/C was deposited at an angle of $45^{\circ}$ to shadow the replicas and $\mathrm{C}$ was deposited at an angle of $90^{\circ}$ to consolidate the replicas. Finally, the resulting replicas were observed using a JEM-100CX electron microscope.

\section{Cryo-transmission electron microscopy (Cryo-TEM)}

The observation was performed using a $120 \mathrm{kV} \mathrm{JEM-2010} \mathrm{Plus}$ TEM instrument, which was equipped with a Gatan cryo Holder 626 and a Gatan US1000 894CCD monitor. First, the samples were stored in a controlled environment vitrification system (CEVS). Then, $5 \mu \mathrm{L}$ of the sample was loaded onto a carbon-coated holey film, which was supported by a copper grid. The sample was immediately plunged into a $-180{ }^{\circ} \mathrm{C}$ liquid ethane reservoir a few seconds later. Finally, it was transferred into liquid nitrogen $\left(-196^{\circ} \mathrm{C}\right)$ for storage until its observation. 


\section{Results and discussion}

\section{Ratio versus EO moieties}

Fig. S1 in the ESI $\uparrow$ displays the phase behaviour of the CTAB/ NPEC- $n$ solutions with CTAB at various proportions. I, II, III and IV represent the regions of the dilute solution, viscoelastic solution, phase separation, and precipitation, respectively. The viscoelastic solutions in region II were studied further.

The relationships between the molar ratio and viscosity of the CTAB/NPEC- $n$ solutions are shown in Fig. $1 ; \alpha_{\text {CTAB }}$ and $\alpha_{\text {NPEC }}$ represent the molar percentage of CTAB and NPEC in the solutions, respectively, while $\alpha_{\mathrm{CTAB}}+\alpha_{\mathrm{NPEC}}=1$. The results show that the viscosities of the separated anionic-nonionic and cationic surfactant solutions were small $(<5 \mathrm{MPa} s)$ at low concentrations. However, except for the CTAB/NPEC-10 solution, the viscosity greatly increases when mixing CTAB with the NPEC- $n$ surfactants together at various molar ratios. Taking the system with a total concentration of $75 \mathrm{mmol} \mathrm{L}^{-1}$ as an example, the highest viscosities of CTAB/NPEC- 5 and CTAB/ NPEC-7 appear at the molar ratio of $76: 24$ and $40: 60$, respectively. This indicates that the EO moieties participate in the synergistic thickening effect and the introduction of EO moieties makes a difference in the optimum mixing ratios. Fig. 2a and b display the aggregate microstructures of the CTAB/ NPEC-5 and CTAB/NPEC-7 solutions in which the $\alpha_{\text {CTAB }}$ values are 0.76 and 0.4 , respectively. In Fig. 2a, spherical aggregates are evenly and sparsely distributed in the CTAB/NPEC-5 solution, and this phenomenon also corresponds to the low viscosity. In Fig. $2 b$, there are diverse aggregates, such as vesicles, rod-like micelles, etc., and they are densely packed, leading to a relatively high viscosity. These findings are in good agreement with those reported in previous studies.

For systems at low concentration and with no additive composition, it is generally believed that most of the aggregates are spherical micelles if the concentration is lower than 10 times the $C_{\mathrm{CMC}}$, whereas vesicles, rod-like micelles, WLMs, and layered structure will appear if the concentration equals or exceeds 10 times the $C_{\mathrm{CMC}} \cdot{ }^{44}$ At this experimental concentration

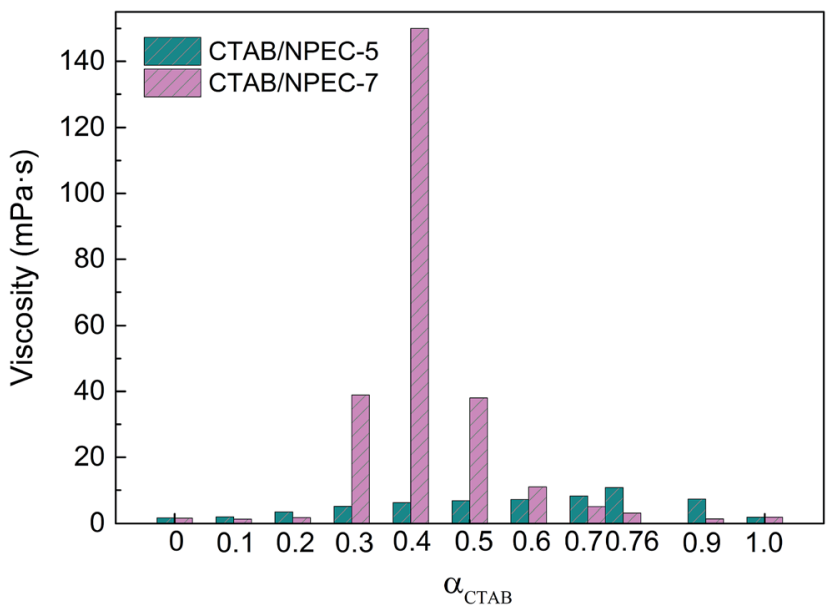

Fig. 1 The viscosity as a function of $\alpha_{C T A B}$ for CTAB/NPEC -5 and CTAB/NPEC-7 solutions (the total concentration was $75 \mathrm{mmol} \mathrm{L}^{-1}$ ).

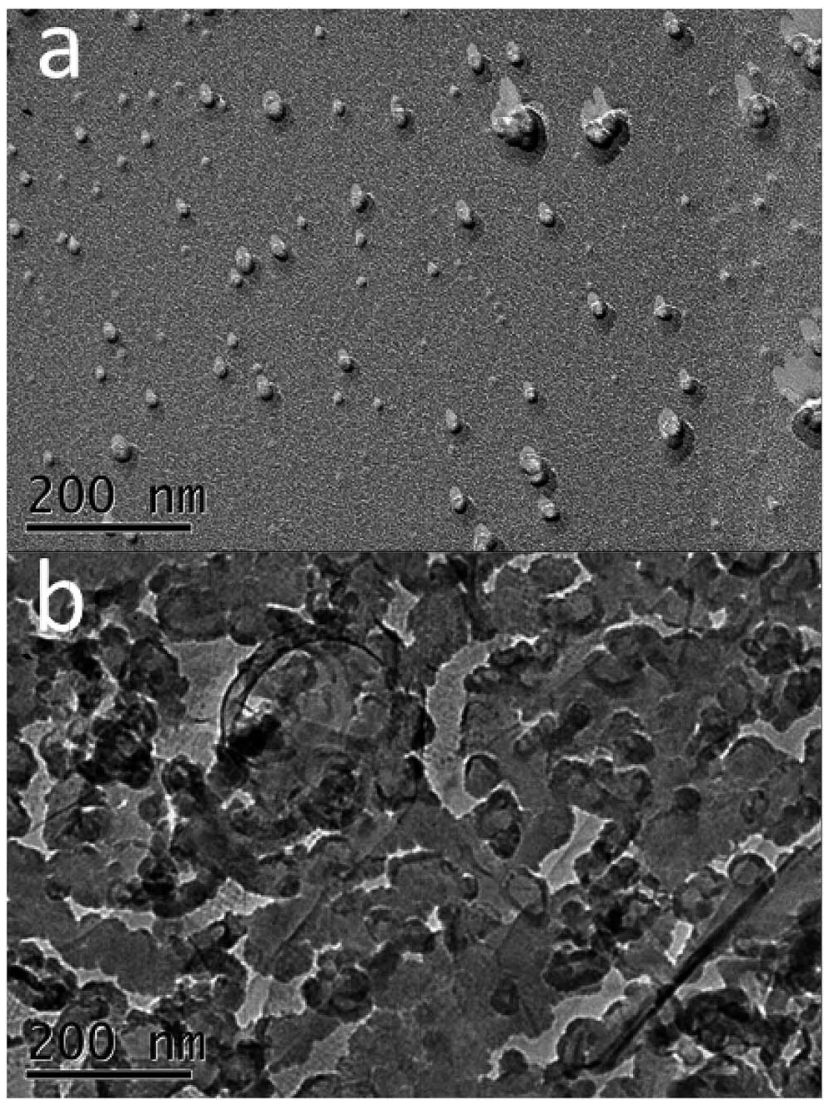

Fig. 2 (a) The images for CTAB/NPEC-5 $\left(\alpha_{\mathrm{CTAB}}=0.76\right)$ and (b) CTAB/ NPEC-7 $\left(\alpha_{\mathrm{CTAB}}=0.4\right)$ with a total concentration of $75 \mathrm{mmol} \mathrm{L}^{-1}$ at $25{ }^{\circ} \mathrm{C}$

(75 $\mathrm{mmol} \mathrm{L}^{-1}$ ) and optimum ratio, the CTAB/NPEC-7 system was susceptible to form non-spherical aggregates including vesicles, rod-like micelles, and WLMs, whereas the CTAB/NPEC5 system did not reach the entanglement concentration (see Fig. 10a). Further explanations have been made with the critical packing parameter $P=V_{\mathrm{C}} /\left(a l_{\mathrm{c}}\right)$, which was proposed by Israelachvili et $a l .{ }^{45} P$ is used for predicting the aggregated structures in aqueous solutions by calculating the geometrical shape of the amphiphilic molecules. In this theory, $V_{\mathrm{C}}$ is the volume of the hydrophobic group of surfactant, $a$ is the area of one headgroup when closely arranged at the aggregate surface, $l_{\mathrm{c}}$ is the chain length of the amphiphilic molecules and the maximum length appears when the hydrophobic chain is fully extended. When the surfactant molecule is conical, $P \leq 1 / 3$, spherical micelles are generally formed. If the molecular structure is similar to a column, $1 / 3<P \leq 1 / 2$, asymmetric micelles are usually formed, including ellipsoid, rod-like micelles or WLMs. When $1 / 2<P \leq 1$, vesicles or layered structures are likely to be formed.

The electrostatic attractions between the positively charged head-group of CTAB and the negatively charged head-group of NPEC- $n$ contribute to the diminishing of distance among the surfactant molecules, leading to various $a$ and $P$ values. The CTAB/NPEC-5 system was prone to form spherical aggregates due to the large head-group at our experimental concentration. 
As the CTAB/NPEC-7 systems reached their entanglement concentration and maximum adsorption (see Fig. 10a), the head-group area was $\sim 0.49 \mathrm{~nm}^{2}$ and the $P$ value was calculated in the following way: the head-group area of the mixture is an average value, $a=\left(a_{\text {cation }}+a_{\text {anion }}\right) / 2$. The total chain volume equals to the sum of two parts, $V_{\mathrm{C}}=V_{\text {cation }}+V_{\text {anion }}$. Moreover, $V_{\text {cation }}, V_{\text {anion }}$, and $l_{\text {c }}$ can be calculated by the following formula according to the carbon number on hydrophobic chain: ${ }^{46} \mathrm{~V}=$ $(27.4+26.9 n) \times 10^{-3} \mathrm{~nm}^{3}, l_{\mathrm{c}}=(0.15+0.1265 n) \mathrm{nm}$. Therefore, $P_{7}$ was calculated to be 0.68 , which is in the range of $1 / 2<P \leq 1$, suggesting the formation of vesicles. However, the EO moieties in the molecular chain of NPEC-7 lead to a larger head group area when the steric hindrance was considered. Thus, the practical $P$ value was smaller than the calculated value. This result was consistent with the coexistence of various aggregates observed in Fig. 2b and the viscosity shown in Fig. 1 and 10a.

\section{Rheological behaviour of the CTAB/NPEC-5 mixtures}

To study the rheological properties of the mixed systems at different concentrations, static shear and oscillation measurements were conducted. Taking the CTAB/NPEC-5 solutions as an example, the molar ratios were fixed at $76: 24\left(\alpha_{\mathrm{CTAB}}=0.76\right)$. As shown in Fig. 3, the solutions behave as Newtonian fluids without viscoelasticity when the total concentration was less than $100 \mathrm{mmol} \mathrm{L}^{-1}$ and as non-Newtonian fluids when the total concentrations were higher than $100 \mathrm{mmol} \mathrm{L}^{-1}$. The viscosity increases upon an increase in the total concentration. This could be interpreted by the following statement. At lower concentrations, the CTAB and NPEC-5 molecules align at the surface. When the concentration is above the CMC, the surfactant molecules assemble into various aggregates such as rod-like micelles, WLMs, etc. presenting a high viscoelastic property. ${ }^{47}$

Furthermore, the viscosity as a function of shear rate $(\gamma)$ for the CTAB/NPEC-5 mixtures at various total concentrations is displayed in Fig. 3. When the concentration was lower than $100 \mathrm{mmol} \mathrm{L}^{-1}$, the viscosity of the solution was rather low and

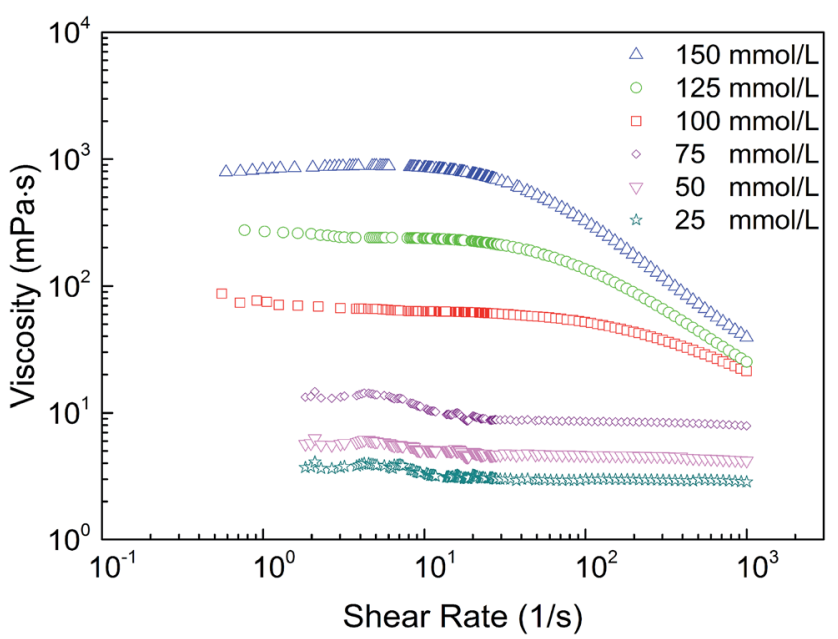

Fig. 3 The steady rheological curves obtained for CTAB/NPEC-5 at various total concentrations. independent of the shear rate. In contrast, when the concentration was above $100 \mathrm{mmol} \mathrm{L}^{-1}$, a Newtonian plateau appears in each solution at low $\gamma$ and shear-thinning behaviour occurs after the critical shear rate $\left(\gamma_{c}\right)$. These are typical properties for WLMs when subjected to a high flow-rate. ${ }^{48}$ The WLMs undergo a quick and repeated destruction-recovery process in the plateau region until the shear rate reaches $\gamma_{c} \cdot{ }^{49,50}$

The zero-shear viscosity of the samples was obtained by extrapolating the Newtonian plateau to $\gamma=0$. It was found that $\eta_{0}$ increases upon increasing the total concentration (see Fig. 10a). The high $\eta_{0}$ value was attributed to the formation of entangled WLMs. When the shear rate was above $\gamma_{c}$, the WLMs do not have enough time to deform in response to the shear stress, which leads to the destruction of the network structure. Accordingly, the viscoelastic solution completely converses into a water-like fluid, exhibiting a shear-thinning behaviour. ${ }^{51,52}$

The Maxwell model, which describes the dynamic rheological properties of viscoelastic materials, is an important criterion for characterizing WLMs. The equations are shown below: ${ }^{53-56}$

$$
\begin{aligned}
& G^{\prime}(\omega)=\frac{G_{\infty}^{\prime}\left(\omega \tau_{\mathrm{R}}\right)^{2}}{1+\left(\omega \tau_{\mathrm{R}}\right)^{2}} \\
& G^{\prime \prime}(\omega)=\frac{G_{\infty}^{\prime}\left(\omega \tau_{\mathrm{R}}\right)}{1+\left(\omega \tau_{\mathrm{R}}\right)^{2}}
\end{aligned}
$$

where $\omega$ is the angular frequency and $G^{\prime}$ and $G^{\prime \prime}$ are the elastic modulus and viscous modulus, respectively. $G^{\prime}$ and $G^{\prime \prime}$ meet at one point, of which the corresponding abscissa is marked as $\omega_{\mathrm{c}}$. The relaxation time $\tau_{\mathrm{R}}$ is defined as the response time of the WLMs to stress, which can be calculated using the following formula: ${ }^{57} \tau_{\mathrm{R}}=1 / \omega_{\mathrm{c}} . G^{\prime}{ }_{\infty}$ is the plateau modulus and equals the maximum value of $G^{\prime}$ at high frequency. However, there may be no platform for some surfactant solutions, which show small deviations from the Maxwell model. In this case, $G^{\prime}{ }_{\infty}$ can be estimated using the following equation $G_{\infty}^{\prime}=2 G^{\prime \prime}{ }_{\max }$, where $G^{\prime \prime}{ }_{\max }$ is the viscous modulus at $\omega_{\mathrm{c}}$.

Oscillatory measurements were conducted to describe the rheological behaviour of the solutions. Fig. 4a shows the variation of the modulus as a function of oscillatory angular frequency $\omega$ for the CTAB/NPEC-5 mixed systems at various total concentrations. At low frequency, the system showed a liquidlike property $\left(G^{\prime \prime}>G^{\prime}\right)$, whereas at high frequency, a solid-like property was observed $\left(G^{\prime \prime}<G^{\prime}\right)$. When the total concentration was higher than $100 \mathrm{mmol} \mathrm{L}^{-1}$, the elastic modulus and the viscous modulus intersected at an exclusive point, i.e. $\omega_{\mathrm{c}}$, rendering a single relaxation time, which is another characteristic of WLMs; ${ }^{58}$ the higher the total concentration, the higher the relaxation time and viscoelasticity.

To investigate how well the rheological data fit the Maxwell model, a Cole-Cole curve $\left(G^{\prime \prime}\right.$ versus $\left.G^{\prime}\right)$ is usually applied. As presented in Fig. $4 \mathrm{~b}$, the solid points refer to the experimental data, whereas the solid line is the fitted data of the samples. When the concentration exceeds $100 \mathrm{mmol} \mathrm{L}^{-1}$, the data can fit well with the semicircle at low frequency. However, it deviates from the Cole-Cole curves at high frequency, which denotes an 

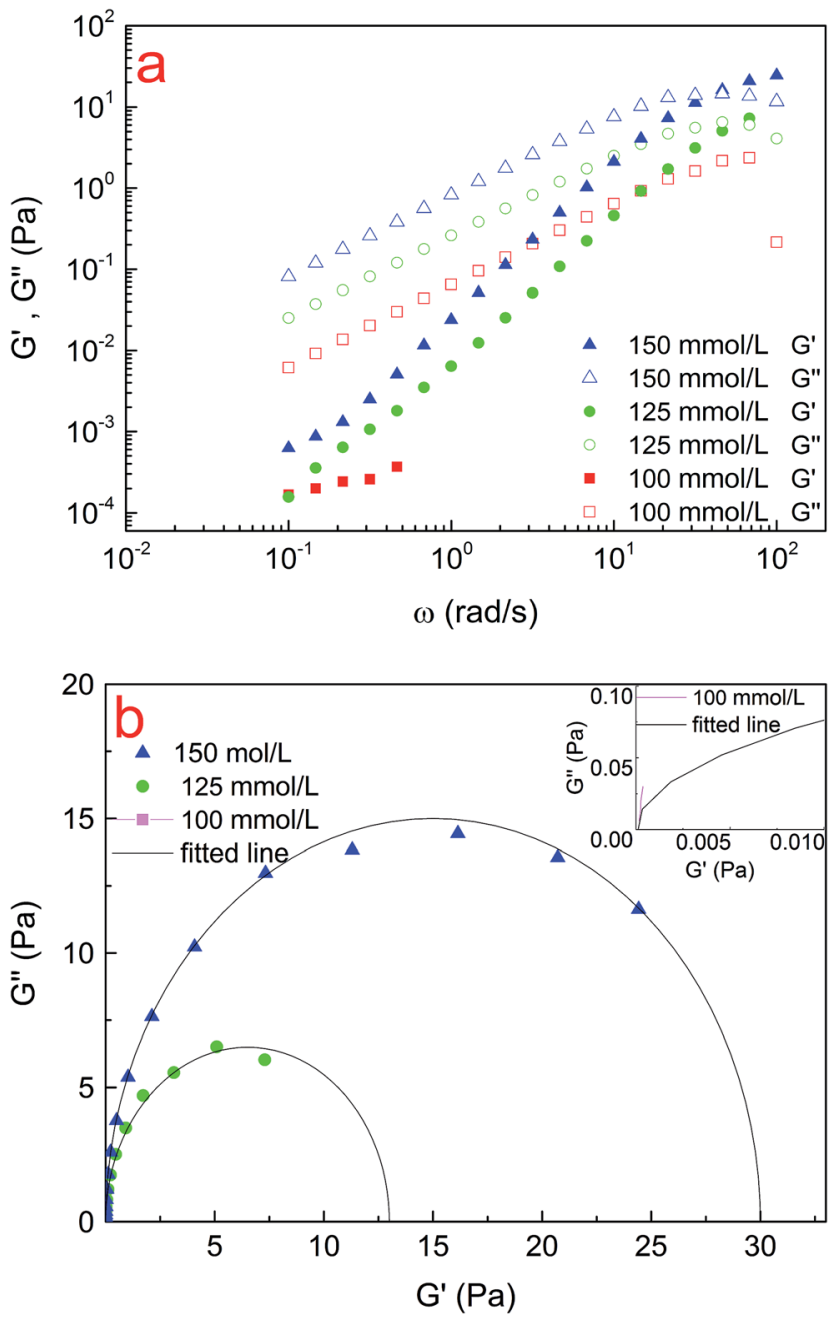

Fig. 4 The (a) dynamic rheological curves and (b) Cole-Cole curves obtained for CTAB/NPEC- 5 with various total concentrations at $25^{\circ} \mathrm{C}$. The inset of (b) is the experimental data and the fitted line of the systems at the total concentration of $100 \mathrm{mmol} \mathrm{L}^{-1}$.

appearance of breathing or Rouse relaxation moles at short time scales. ${ }^{59}$ In addition, when the total concentration increases above the entangled concentration, the surfactant molecules more closely self-assemble and thus the aggregates tend to rapidly grow into WLMs, as verified in Fig. 2a and 5. Moreover, the spherical aggregates transform into long entangled WLMs.

\section{The effect of $\mathrm{NaCl}$ on the rheological behaviour of the CTAB/ NPEC-5 mixtures}

The repulsion between head groups with like charges will hinder the micellization. Upon the addition of $\mathrm{NaCl}$, the charge repulsion was screened. This process resulted in a smaller distance between these micelles, ${ }^{60}$ leading to various rheological behaviours in the mixed solutions. ${ }^{61}$ The viscosity, viscoelasticity, and shear-resistance of CTAB/NPEC-5 with NaCl were investigated and the effect of $\mathrm{NaCl}$ was analyzed in detail. Fig. S2 in the ESI $\dagger$ shows the viscosity as a function of shear rate at various $\mathrm{NaCl}$ concentrations for the CTAB/NPEC-5 systems

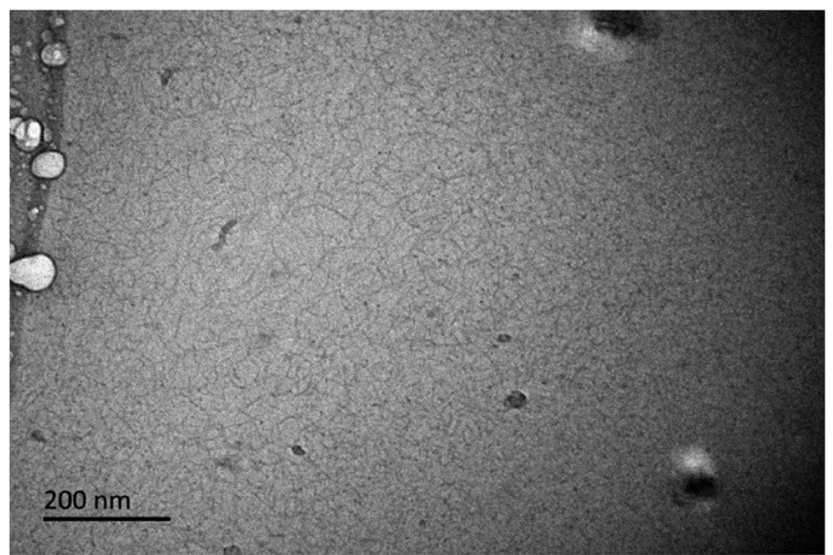

Fig. 5 A Cryo-TEM image obtained for CTAB/NPEC-5 without salt at the total concentration of $150 \mathrm{mmol} \mathrm{L}^{-1}$.

with a fixed molar ratio of $76: 24$, total concentration of $150 \mathrm{mmol} \mathrm{L}^{-1}$, and at the temperature of $25^{\circ} \mathrm{C}$. All the samples exhibited a Newtonian plateau at low $\gamma$ and shear-thinning behaviour when the shear rates were above $\gamma_{\mathrm{c}}$. This phenomenon can be explained by Rheo-SANS (Rheo-Small Angle Neutron Scattering) measurements. ${ }^{62,63}$ When the shear rate reaches $\gamma_{c}$, the WLMs are inclined to arrange themselves along the direction of shearing, i.e. no entanglement among the WLMs, and thus, a reduction in the viscosity occurs. Moreover, the viscosity increases at first and then drops, as shown in Fig. 6. The transition of viscosity occurred when the $\mathrm{NaCl}$ concentration was $2565 \mathrm{mmol} \mathrm{L}^{-1}$. This may be attributed to the transformation from linear WLMs to branched WLMs. Phase separation appeared at $3420 \mathrm{mmol} \mathrm{L}^{-1} \mathrm{NaCl}$ due to the formation of aggregates with various sizes. The electrostatic attraction between the positive charges of the anionic-nonionic surfactants and negative charges of the cationic surfactants facilitated the formation of aggregates via self-assembly. In addition, there were electrostatic repulsions between the

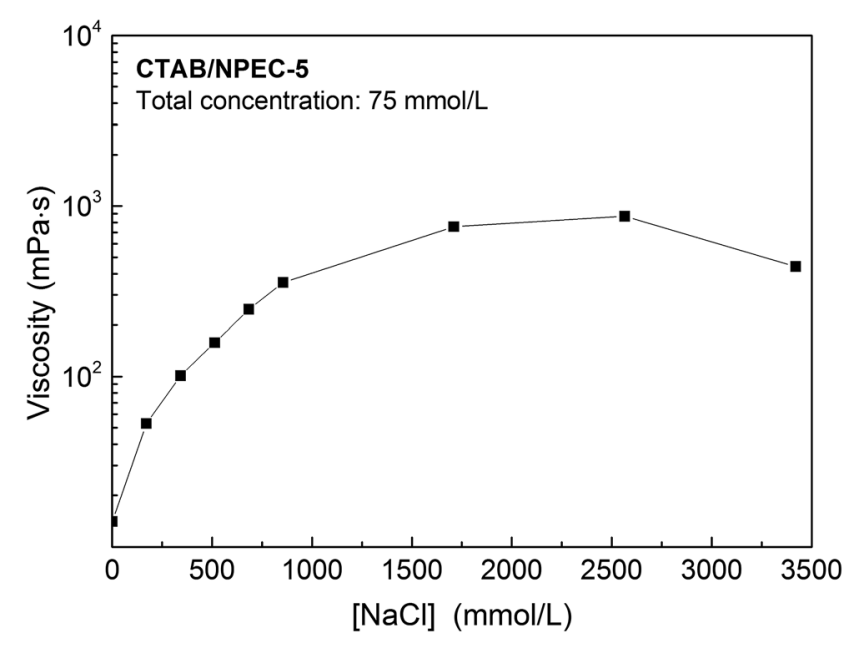

Fig. 6 The viscosity as a function of the molar concentration of $\mathrm{NaCl}$ for CTAB/NPEC-5 (total concentration is $75 \mathrm{mmol} \mathrm{L}^{-1}$ and the shear rate is $2.5 \mathrm{~s}^{-1}$ ). 
molecules with like charges. When the counter-ions were added to the solution, the electrostatic repulsion between the headgroups with like charges were screened. The reduction of the repulsion led to a decreased area of the head-group and an increased $P$ value. This phenomenon suggests that the micelles will quickly grow along one dimension. Thus, it leads to a sharp increase in the viscosity before the $\mathrm{NaCl}$ concentration reaches $2565 \mathrm{mmol} \mathrm{L}^{-1}$. Once the branching behaviour of the entangled WLMs occurs, the viscosity will gradually decrease (see Fig. 6).

The effects of $\mathrm{NaCl}$ concentration on the dynamic oscillation measurements of CTAB/NPEC-5 are illustrated in Fig. S3a (see the ESI $\dagger$ ). Except for the mixed solutions with $171 \mathrm{mmol} \mathrm{L}^{-1}$ $\mathrm{NaCl}$, the elastic modulus and the viscous modulus meet at one point, indicating the presence of a single relaxation time and the formation of WLMs. Fig. S3b in the ESI $\dagger$ depicts the ColeCole plots of the samples with different $\mathrm{NaCl}$ concentrations. When the $\mathrm{NaCl}$ concentration was higher than $171 \mathrm{mmol} \mathrm{L}^{-1}$, it can be seen that the experimental data can fit well with the theoretical value at low frequency, which is consistent with the previous finding related to the formation of WLMs.

$\tau_{\mathrm{R}}$ and $G_{\infty}^{\prime}$ as a function of $\mathrm{NaCl}$ concentration are presented in Fig. S4 (ESI, $\dagger$ see line a and line b). As is known, $\tau_{\mathrm{R}}$ and $G_{\infty}^{\prime}$ are usually applied to characterize the viscosity and elasticity of the systems, respectively. As seen in Fig. S4 (see the ESI†े), $G_{\infty}^{\prime}$ monotonically increases with the $\mathrm{NaCl}$ concentration. This indicates that more entangled WLMs intertwined with each

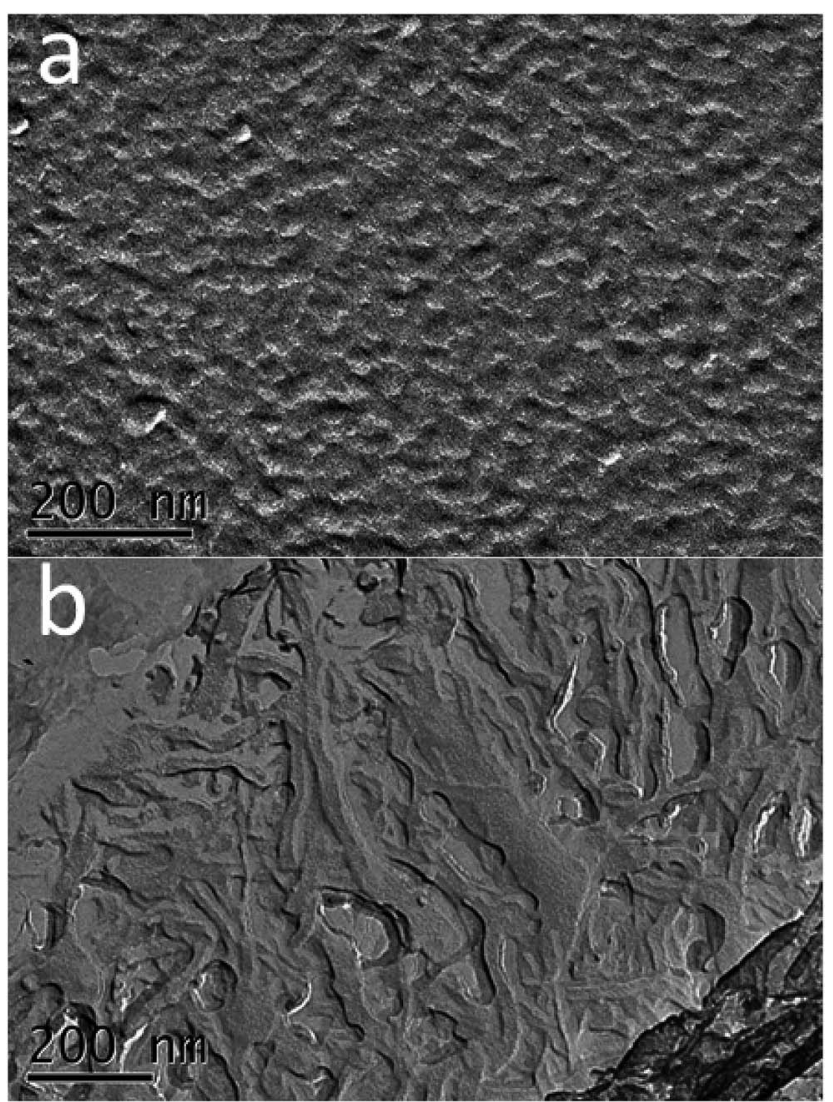

Fig. 7 Images of CTAB/NPEC-5 (75 $\mathrm{mmol} \mathrm{L}^{-1}, 76$ : 24) with $171 \mathrm{mmol}$ $\mathrm{L}^{-1}$ (a) and $855 \mathrm{mmol} \mathrm{L}^{-1}$ (b) $\mathrm{NaCl}$ at $25^{\circ} \mathrm{C}$. other, i.e. the degree of the entanglement increases. In contrast with $G_{\infty}^{\prime}$, when the $\mathrm{NaCl}$ concentration was lower than $2565 \mathrm{mmol} \mathrm{L}^{-1}$, the $\tau_{\mathrm{R}}$ value increases, leading to an extension of the WLMs in one dimension and an increase in viscosity. The tendency of the change in $\tau_{\mathrm{R}}$ was consistent with that of viscosity; both of them decreased when the $\mathrm{NaCl}$ concentration reached $2565 \mathrm{mmol} \mathrm{L}^{-1}$, indicating that the relaxation process was relevant to the length of WLMs. Taking $\tau_{\mathrm{R}}$ and $G_{\infty}^{\prime}$ into consideration, it is clear that the viscosity decreases due to the branching of the micelles.

These results are in agreement with that reported by Pei et al. ${ }^{49}$ In spite of branching, the micelles can still entangle with each other and form a network; hence, the elasticity increases. Fig. 7a and $b$ show the microstructures of CTAB/NPEC-5 with $171 \mathrm{mmol} \mathrm{L}^{-1}$ and $855 \mathrm{mmol} \mathrm{L}^{-1} \mathrm{NaCl}$. At a relatively low $\mathrm{NaCl}$ concentration, vesicles and rod-like micelles appear; at a relatively high $\mathrm{NaCl}$ concentration, WLMs are observed, making the solution a viscoelastic fluid. In conclusion, $\mathrm{NaCl}$ promotes the growth of aggregates to some extent.

\section{Shearing resistance of the CTAB/NPEC-5 mixtures with salt}

The addition of $\mathrm{NaCl}$ to a CTAB/NPEC-5 solution promotes the conversion of the aggregates, which indirectly affects the rheological behaviour and shear-resistance. Fig. 8 shows the viscosity as a function of shearing time. The viscosity of CTAB/ NPEC-5 with salt remains almost constant under a shear rate of $170 \mathrm{~s}^{-1}$ for $30 \mathrm{~min}$. It was indicated that the system still possesses high viscosity and a good shear-resistance in spite of the addition of salt. This is analogous to the case of the salt effect on the viscosity (see Fig. 6).

The static screening effect of the counter-ions was beneficial to the growth of the micelles. Entangled WLMs enable the formation of the network and make the system more structured, which results in a better viscoelasticity and shear-resistance in the solution. Fig. $2 \mathrm{a}$ and $7 \mathrm{~b}$ are the images before and after the addition of $855 \mathrm{mmol} \mathrm{L}^{-1} \mathrm{NaCl}$. In Fig. 2a, there is a large

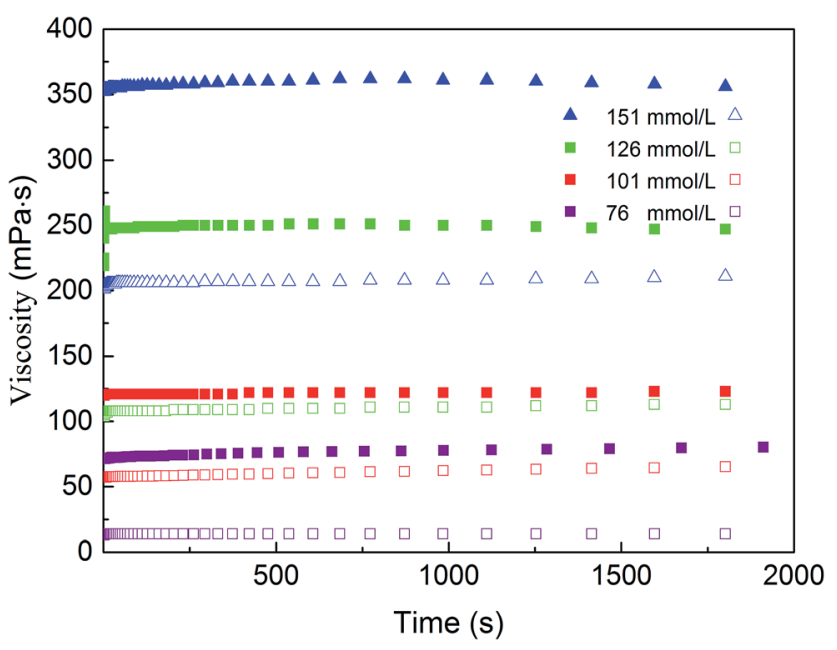

Fig. 8 Viscosity as a function of shearing time for CTAB/NPEC-5 with various concentrations of $\mathrm{NaCl}$ (solid symbol: with $\mathrm{NaCl}$; open symbol: without $\mathrm{NaCl}$; shear rate, $170 \mathrm{~s}^{-1}$ ). 
quantity of spherical aggregates, whereas in Fig. 7b, entangled WLMs can be observed. By combining the rheological behaviour, the viscosity, and viscoelasticity, the shear-resistance can be explained. It is obvious that the formation of spherical aggregates enables low viscosity and viscoelasticity in the sample (see Fig. 2a and 10a). After adding $855 \mathrm{mmol} \mathrm{L}^{-1} \mathrm{NaCl}$, the spherical aggregates quickly grow into the entangled WLMs (see Fig. 7b), forming a tight network structure. Consequently, the saturated network endows the solution with a good shearresistance and the solution behaves as a non-Newtonian fluid.

\section{Thermal-sensitivity versus the EO moieties}

The viscosity and transmittance as a function of temperature for the mixed systems are illustrated in Fig. 9a and b, respectively. When the temperature was below $35^{\circ} \mathrm{C}$, the viscosities of CTAB/ NPEC-7, CTAB/NPEC-5 without NaCl, and CTAB/NPEC-5 with $\mathrm{NaCl}$ gradually decreased (Fig. 9a). However, the transmittance of three systems rapidly increased before $30{ }^{\circ} \mathrm{C}$ and then remained almost constant between 30 and $35^{\circ} \mathrm{C}$ (Fig. 9b). This
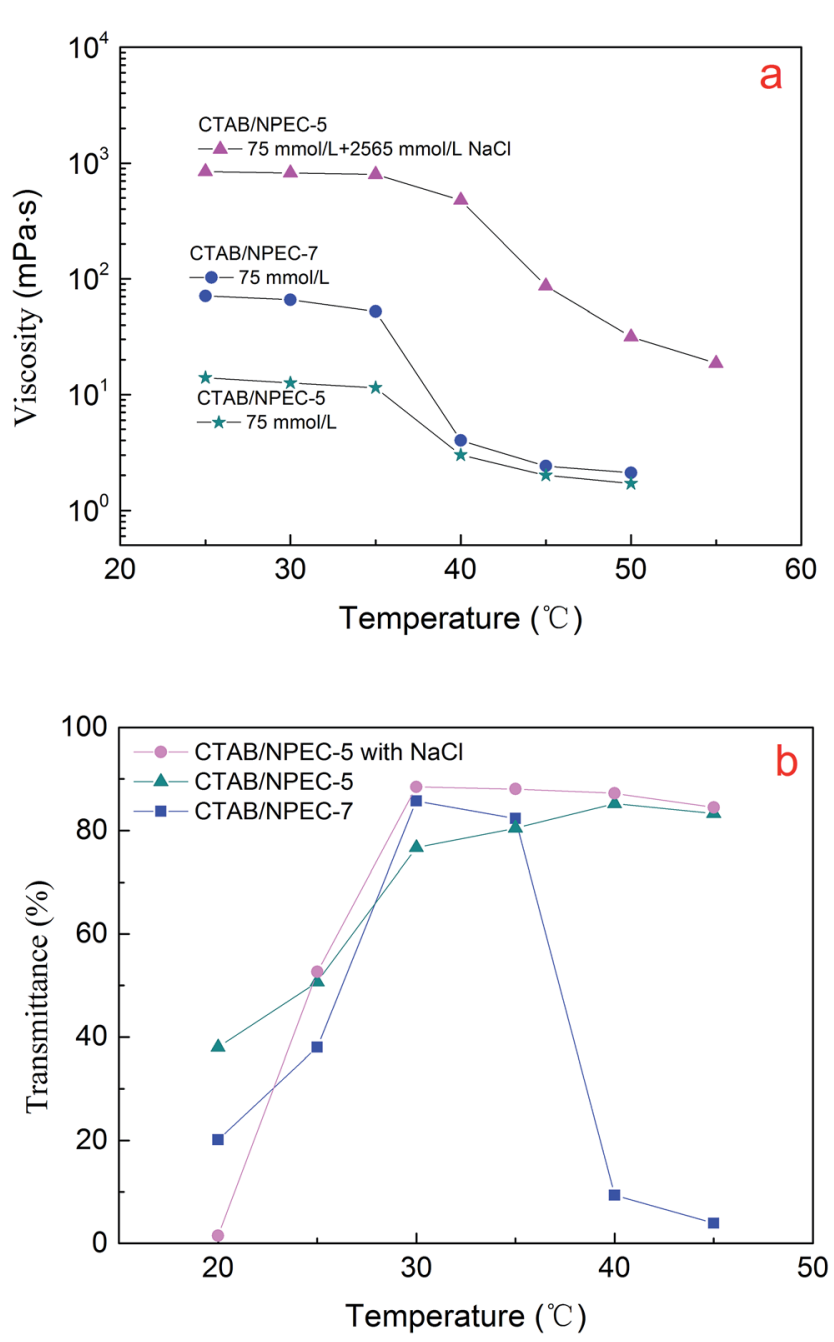

Fig. 9 Viscosity (a) (shear rate, $6 \mathrm{~s}^{-1}$ ) and transmittance (b) as a function of temperature for the bicomponent surfactants (the total concentration of all the mixtures is $75 \mathrm{mmol} \mathrm{L}^{-1}$ ). means before the critical temperature $\left(T_{\mathrm{c}}\right)$, the aggregates in the solutions gradually change. Once the temperature reaches $T_{\mathrm{c}}$ $\left(35^{\circ} \mathrm{C}\right)$, significant changes occur. Thus, all the viscosities of the three systems sharply decrease. Furthermore, the transmittance of the CTAB/NPEC-7 solution shows a sharp decrease and the solution becomes turbid, which are in accordance with the reduction in viscosity. The transmittance of CTAB/NPEC-5 with $\mathrm{NaCl}$ continues to slowly decrease, whereas an increase in the transmittance of CTAB/NPEC-5 without $\mathrm{NaCl}$ occurs and no cloudy phenomenon was observed, albeit the temperature reached $45{ }^{\circ} \mathrm{C}$. On the one hand, water increases with temperature due to the enhancement of hydration on the surface of the aggregates. ${ }^{64}$ Therefore, the systems with more EO moieties possess more hydrated water and thus a higher viscosity and better temperature resistance. On the other hand, because the thermal motion speeds up with an increase in temperature, the electrostatic interactions between the molecules are weakened. Correspondingly, the area of the head-group enlarges, the $P$ value diminishes, and the aggregates convert into spherical micelles for all the systems at the experimental concentration. In addition, the temperature-resistant property of the CTAB/ NPEC-5 with NaCl system improves to some degree (see Fig. 9a).

\section{The mechanism of the rheological properties of the mixtures with various EO moieties}

The viscosities of CTAB/NPEC-5, CTAB/NPEC-7, and CTAB/ NPEC-10 were studied to investigate the effect of the EO moieties on the rheological behaviour.

For CTAB/NPEC-10, there are 10 EO moieties in the hydrophobic chain of NPEC-10, and the viscosity remains almost constant and behaves like water even when the concentration reaches $150 \mathrm{mmol} \mathrm{L}^{-1}$ (see Fig. 10a). It is clear that the $10 \mathrm{EO}$ moieties were not well aligned anymore. The molecular conformation results in larger steric hindrance than those of NPEC-5 and NPEC-7, resulting in an increased area of the headgroup. Thus, the CTAB and NPEC-10 molecules tend to form micelles with small size instead of assembling into long micelles.

For CTAB/NPEC-7, the viscosity gradually increases with the total concentration. There are $7 \mathrm{EO}$ moieties in the hydrophobic chain of NPEC-7. The conformation of the NPEC-7 molecule is similar to that of NPEC-10. However, the steric hindrance of NPEC-7 is smaller due to less EO moieties and so is the area of the head-group. Therefore, spherical micelles were no longer the only aggregates in the solution. That is the reason for the higher viscosity of CTAB/NPEC-7 when compared to that of the CTAB/NPEC-10 systems.

For CTAB/NPEC-5, the viscosity can increase three orders of magnitude as the concentration increases up to $150 \mathrm{mmol} \mathrm{L}^{-1}$. Once the total concentration exceeds the micellar entanglement value, the viscosity increases significantly and a large quantity of non-spherical aggregates appear. The morphologies shown in Fig. 2a and 5 display the transformation of the aggregates. When the total concentration was $75 \mathrm{mmol} \mathrm{L}^{-1}$, spherical aggregates with uniform size were sparsely dispersed in the solution (see Fig. 2a). In the solution at the concentration of 

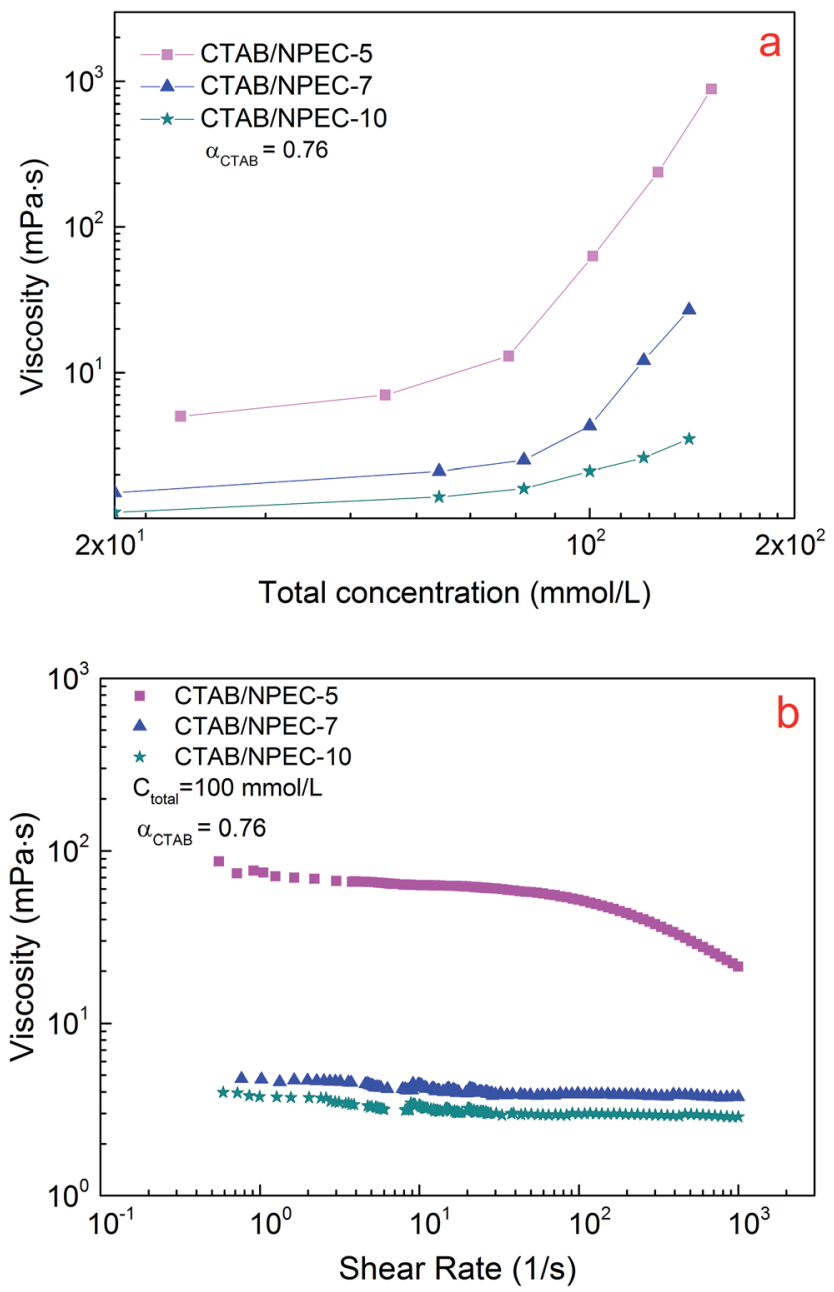

Fig. 10 The viscosity of the solutions as a function of the total concentration (a) (shear rate, $6 \mathrm{~s}^{-1}$ ) and (b) the shear rate for the systems with various $\mathrm{EO}$ numbers.

$150 \mathrm{mmol} \mathrm{L}^{-1}$, entangled WLMs mostly intertwined with each other and formed a network (see Fig. 5), which corresponds to the high viscoelasticity. When compared with NPEC-7, there are 5 EO moieties in the hydrophobic chain of NPEC-5 and the lower number of EO moieties leads to a smaller area of the head-group. Thus, a tighter arrangement among the molecules occurred, facilitating the growth of the aggregates along one dimension before its maximum length and then the viscosity greatly increased.

Our study reveals that an appropriate number of EO moieties contribute to the formation of the high viscoelastic fluids and satisfy practical applications well.

\section{Conclusion}

The number of EO moieties contribute to the diverse conformations of EO chains and the morphologies of the aggregates, leading to various molar ratios and rheological behaviours among CTAB/NPEC- $n$. The conformations of the EO moieties concern the head-group area and steric hindrance, which affect the arrangement of the surfactant molecules. The anionicnonionic surfactant with 5 EO moieties and 7 EO moieties can form entangled WLMs, which enables a higher viscoelasticity in the mixtures under an appropriate external environment. Upon increasing the total concentration, the aggregates transform from spherical micelles to vesicles, WLMs, and other micelles. Accordingly, the viscoelasticity of the solution varies. The viscosities and transmittance of the CTAB/NPEC-5 and CTAB/ NPEC-7 solutions drop at the transition temperature of $35{ }^{\circ} \mathrm{C}$. $\mathrm{NaCl}$ facilitates the formation of entangled WLMs and networks, leading to an increase in the viscosity. A high $\mathrm{NaCl}$ concentration prompts an increase in the elasticity, enabling a good shear-resistance for the network. However, the viscosity decreases when precipitation occurs at a high NaCl concentration $\left(\sim 3520 \mathrm{mmol} \mathrm{L}^{-1}\right)$. This study has extended the understanding of rheological behaviour of anionic-nonionic carboxylate/cationic surfactant systems as well as the effect of the EO moieties. The EO moieties play an important role in the formation of aggregates and the rheological behaviour can be effectively regulated by introducing EO moieties with appropriate numbers.

\section{Acknowledgements}

This work was financially by the National Natural Science Foundation of China (No. 21273286, 51574267) and Science Foundation of China University of Petroleum, Beijing (No. 2462015YJRC033).

\section{Notes and references}

1 P. Parekh, D. Varade, J. Parikh and P. Bahadur, Colloids Surf., A, 2011, 385, 111-120.

2 V. B. Fainerman, R. Miller and E. V. Aksenenko, Adv. Colloid Interface Sci., 2002, 96, 295.

3 S. Ghosh, J. Colloid Interface Sci., 2001, 244, 128-138.

4 C. Das, T. Chakraborty, S. Ghosh and B. Das, Colloid J., 2010, 72, 788-798.

5 D. Tikariha, B. Kumar, S. Ghosh, A. K. Tiwari, S. K. Saha, N. Barbero, P. Quagliotto and K. K. Ghosh, J. Nanofluids, 2013, 2, 316-324.

6 P. K. Khatua, S. Ghosh, S. K. Ghosh and S. C. Bhattacharya, J. Dispersion Sci. Technol., 2005, 25, 741-748.

7 P. Sharma, S. Sachar, G. Kaur, P. Thakur, S. B. Mandeep and T. S. Banipal, J. Surf. Sci. Technol., 2007, 23, 131-147.

8 M. Prasad, A. M. Donald, R. Palepu and S. P. Moulik, J. Surf. Sci. Technol., 2003, 19, 125-138.

9 H. Oda, S. Nagadome, S. Lee, F. Ohseto, Y. Sasaki and G. Sugihara, J. Surf. Sci. Technol., 1998, 14, 1-22.

10 S. K. Suri and H. S. Randhawa, J. Surf. Sci. Technol., 1989, 5, 355-363.

11 S. B. Sulthana, S. G. T. Bhat and A. K. Rakshit, J. Surf. Sci. Technol., 1997, 13, 20-31.

12 P. Brown, C. P. Butts and J. Eastoe, Soft Matter, 2013, 9, 2365. 13 S. W. Liu, X. W. Wang, L. M. Chen, L. X. Hou and T. H Zhou, Soft Matter, 2014, 10, 9177-9186.

14 G. Palazzo, Soft Matter, 2013, 9, 10668. 
15 C. A. Dreiss, Soft Matter, 2007, 3, 956-970.

16 T. Chakraborty and S. Ghosh, J. Surfactants Deterg., 2008, 11, 323-334.

17 R. Angelico, S. Amin, M. Monduzzi, S. Murgia, U. Olsson and G. Palazzo, Soft Matter, 2012, 8, 10941.

18 A. D. Wang, W. Y. Shi, J. B. Huang and Y. Yan, Soft Matter, 2016, 12, 337-357.

19 H. F. Shi, W. Ge, H. Oh, S. M. Pattison, J. T. Huggins, Y. Talmon, D. J. Hart, S. R. Raghavan and J. L. Zakin, Langmuir, 2013, 29, 102-109.

20 N. Hassan, J. M. Ruso and A. González-Pérez, Soft Matter, 2011, 7, 5194.

21 S. Ghosh, A. Das Burman, G. C. De and A. R. Das, J. Phys. Chem. B, 2011, 115, 11098-11112.

22 S. Ghosh, G. B. Ray and S. Mondal, Fluid Phase Equilib., 2015, 405, 46-54.

23 S. Das, S. Maiti and S. Ghosh, RSC Adv., 2014, 4, 1227512286.

24 H. Afifi, G. Karlsson, R. K. Heenan and C. A. Dreiss, Langmuir, 2011, 27, 7480-7492.

25 L. S. Hao, P. Hu and Y. Q. Nan, Colloids Surf., A, 2010, 361, 187-195.

26 Y. Y. Lin, X. H. Cheng, Y. Qiao, C. L. Yu, Z. B. Li, Y. Yan and J. B. Huang, Soft Matter, 2010, 6, 902-908.

27 E. F. Marques, R. O. Brito, S. G. Silva, J. E. Rodríguez-Borges, M. L. D. Vale, P. Gomes and O. Söderman, Langmuir, 2008, 24, 11009-11017.

28 H. Q. Yin, Y. Y. Lin, J. B. Huang and J. P. Ye, Langmuir, 2007, 23, 4225-4230.

29 Y. Q. Nan, H. L. Liu and Y. Hu, Colloids Surf., A, 2005, 269, 101-111.

30 J. Mata, D. Varade, G. Ghosh and P. Bahadur, Colloids Surf., A, 2004, 245, 69-73.

31 D. Varade, T. Joshi, V. K. Aswal, P. S. Goyal, P. A. Hassan and P. Bahadur, Colloids Surf., A, 2005, 259, 103-109.

32 X. Chen, T. J. Young, M. Sarkari, R. O. Williams III and K. P. Johnston, Int. J. Pharm., 2002, 242, 3-14.

33 M. Á. Valenzuela, M. P. Gárate and A. F. Olea, Colloids Surf., A., 2007, 307, 28-34.

34 A. Mehreteab and F. J. Loprest, J. Colloid Interface Sci., 1988, 125, 602-609.

35 Y. J. Chen and G. Y. Xu, Colloids Surf., A, 2013, 424, 26-32. 36 M. Hato and K. Shinoda, J. Phys. Chem., 1973, 77, 378-381.

37 R. Varadaraj, J. Bock, P. Geissler, S. Zushma, N. Brons and T. Colletti, J. Colloid Interface Sci., 1991, 147, 396-402.

38 M. Dahanayake, A. W. Cohen and M. J. Rosen, J. Phys. Chem., 1986, 90, 2413-2418.

39 Y. F. Wang and J. B. Huang, Acta Phys.-Chim. Sin., 2001, 488490.
40 C. Minero, E. Pramauro, E. Pelizzetti, V. Degiorgio and M. Corti, J. Phys. Chem., 1986, 90, 1620-1625.

41 A. Masuyama, T. Kawano, Y. Zhu and Y. N. Toshiyuki Kida, Chem. Lett., 1993, 22, 2053-2056.

42 Z. X. Chen, S. P. Deng and X. K. Li, J. Colloid Interface Sci., 2008, 318, 389-396.

43 Z. Y. Liu, L. Zhang, X. L. Cao, X. W. Song, Z. Q. Jin, L. Zhang and S. Zhao, Energy Fuels, 2013, 27, 3122-3129.

44 Z. X. Cheng, X. B. Dong, Q. Y. Pan, J. C. Zhang and X. W. Dong, Mater. Lett., 2006, 60, 3137-3140.

45 J. N. Israelachvili, D. J. Mitchell and B. W. Ninham, J. Chem. Soc., 1976, 72, 1525-1568.

46 J. M. Corkill, J. F. Goodman, C. P. Ogden and J. R. Tate, Proc. R. Soc. A, 1963, 273, 84-102.

47 B. A. Schubert, N. J. Wagner, E. W. Kaler and S. R. Raghavan, Langmuir, 2004, 20, 3564-3573.

48 X. M. Pei, J. X. Zhao, Y. Z. Ye, Y. You and X. L. Wei, Soft Matter, 2011, 7, 2953-2960.

49 X. M. Pei, Z. H. Xu, B. L. Song, Z. G. Cui and J. X. Zhao, Colloids Surf., A, 2014, 443, 508-514.

50 J. H. Huang, S. X. Zhang, Y. H. Feng, J. C. Li, H. Q. Yan, F. R. He, G. Z. Wang, Y. F. Liu and L. N. Wang, Colloids Surf., A, 2016, 500, 222-229.

51 Y. X. Han, Y. J. Feng, H. Q. Sun, Z. Q. Li, Y. G. Han and H. Y. Wang, J. Phys. Chem. B, 2011, 115, 6893-6902.

52 A. A. Dar, A. Garai, A. R. Das and S. Ghosh, J. Phys. Chem. A, 2010, 114, 5083-5091.

53 D. Wang, R. H. Dong, P. F. Long and J. C. Hao, Soft Matter, 2011, 7, 10713-10719.

54 D. P. Acharya, D. Varade and K. Aramaki, J. Colloid Interface Sci., 2007, 315, 330-336.

55 M. E. Cates, Macromolecules, 1987, 20, 2289-2296.

56 S. R. Raghavan and E. W. Kaler, Langmuir, 2001, 17, 300-306.

57 C. Oelschlaeger, P. Suwita and N. Willenbacher, Langmuir, 2010, 26, 7045-7053.

58 F. Chen, Y. Wu, M. Wang and R. L. Zha, Colloid Polym. Sci., 2015, 293, 687-697.

59 A. Parker and W. Fieber, Soft Matter, 2013, 9, 1203-1213.

60 D. F. Yu, X. Huang, M. L. Deng, Y. Y. Lin, L. X. Jiang, J. B. Huang and Y. L. Wang, J. Phys. Chem. B, 2010, 114, 14955-14964.

61 T. Lu, L. G. Xia, X. D. Wang, A. Q. Wang and T. Zhang, Langmuir, 2011, 27, 9815-9822.

62 S. Förster, M. Konrad and P. Lindner, Phys. Rev. Lett., 2005, 94, 017803.

63 R. A. Araujo, D. J. B. Villegas, R. G. Larson and U. M. Cordova-Figueroa, Soft Matter, 2016, 12, 4071-4081.

64 F. N. Padia, M. Yaseen, B. Gore, S. Rogers, G. Bell and J. R. Lu, J. Phys. Chem. B, 2013, 118, 179-188. 